\title{
Interplay of gravitation and linear superposition of different mass eigenstates
}

\author{
D. V. Ahluwalia \\ Physics (P-25) Division, Mail Stop H-846, Los Alamos National Laboratory, Los Alamos, New Mexico 87545 \\ and Global Power Division, ANSER, S-800, 1215 Jefferson Davis Highway, Arlington, Virginia 22202 \\ C. Burgard \\ CERN, PPE, CH-1211 Geneva 23, Switzerland \\ (Received 5 March 1996; revised manuscript received 31 October 1997; published 3 March 1998)
}

\begin{abstract}
The interplay of gravitation and the quantum-mechanical principle of linear superposition induces a new set of neutrino oscillation phases. These ensure that the flavor-oscillation clocks, inherent in the phenomenon of neutrino oscillations, redshift precisely as required by Einstein's theory of gravitation. The physical observability of these phases in the context of the solar neutrino anomaly, type-II supernova, and certain atomic systems is briefly discussed. [S0556-2821(98)00808-X]

PACS number(s): 04.20.Cv, 03.65.Bz, 14.60.Pq
\end{abstract}

\section{THE CONCEPTUAL FRAMEWORK}

To explain recent atmospheric and solar neutrino data the possibility of the violation of the equivalence principle has been considered [1]. It is therefore an important matter to understand in detail how quantum mechanics and gravity work together for neutrino oscillations. In addition, the problem is of interest in its own right to understand explicitly, and in detail, how the principle of equivalence and the principle of linear superposition of quantum mechanics intermingle.

The classical effects of gravitation on a single mass eigenstate are usually considered in terms of a force $\boldsymbol{F}$, whereas the quantum-mechanical ones are determined by the gravitational interaction energy. The interaction energy (denoted by $\left.U_{i n t}^{n r}\right)$ for a nonrelativistic particle of mass $m$ is obtained in the weak-field limit of Einstein's theory of gravitation to have the same form as in Newtonian theory [2]. It reads $U_{i n t}^{n r}=m \phi$, with $\phi=-G M / r$ standing for the gravitational potential of a nonrotating object of mass $M$, while $\boldsymbol{F}=-m \nabla \phi$. The interaction energy for a relativistic particle $U_{i n t}^{\text {rel }}$ in the weak-field limit is more generally obtained from the force $\boldsymbol{F}$ acting on the mass eigenstate $m$ in the Schwarzschild gravitational environment of a mass $M[3]$ :

$$
\boldsymbol{F}=-\frac{G M m \gamma}{r^{3}}\left[\left(1+\beta^{2}\right) \boldsymbol{r}-(\boldsymbol{r} \cdot \boldsymbol{\beta}) \boldsymbol{\beta}\right]
$$

where $\beta=|v| / c, \boldsymbol{v}$ is the velocity of the mass eigenstate considered, and $\gamma=\left(1-\beta^{2}\right)^{-1 / 2}$. Assuming the mass eigenstate to be relativistic and setting $m \gamma=E / c^{2}$ one is led to the expression

$$
U_{i n t}^{r e l}=\int_{\infty}^{r} \boldsymbol{F} \cdot d \boldsymbol{r}^{\prime}=-\frac{G M E}{r c^{2}}=-\left(E / c^{2}\right) \phi .
$$

In order to avoid any possible confusion we wish to quote Okun explicitly (see [4], p. 149): “It is common knowledge that in a locally inertial frame the gravitational force is zero. That means that [Eq. (1)] is valid only for locally [noniner- tial] frames, such as the usual laboratory frame.' Now, along an equipotential surface the force $\boldsymbol{F}$ is known to vanish and consequently no classical effects can be observed in this direction. Indeed, in going to an appropriately accelerated frame the constant potential appearing along a segment of an equipotential surface can be removed.

To contrast these well-known textbook statements [2-5] we wish to focus in the following on some not always fully appreciated aspects regarding the phase of a quantummechanical state. A single nonrelativistic mass eigenstate, considered in an appropriately small region of space-time, picks up an unobservable global phase factor $\exp \left(-i m c^{2} t / \hbar-i m \phi t / \hbar\right)$. In the case of a linear superposition of $n$ eigenstates with different masses $\left(m_{i}, i=1, \ldots, n\right)$, their relative phases turn out to be modified through the gravitation by the mass-dependent factors $\exp \left(-i m_{i} \phi t / \hbar\right)$. We will show below, with appropriate modifications required for neutrinos, that these gravitationally induced relative phases are observable by a distant observer (for local clocks also a redshift) as the redshift of the flavor-oscillation clocks. To be more specific, during the quantum evolution of a linear superposition of mass eigenstates the corresponding gravitationally induced relative phases do not vanish along an equipotential surface even if the gravitational force $\boldsymbol{F}$ does.

For the case considered,

$$
\varphi^{G}=\Phi \varphi^{0}
$$

where $\Phi=\phi / c^{2}$ is the dimensionless gravitational potential, whereas $\varphi^{G}$ and $\varphi^{0}$ stand for the (time-)oscillatory phases with and without gravity, respectively.

For neutrino oscillations the gravitationally induced phase (denoted by $\varphi_{j i}^{G}$ ) comes in addition to the corresponding oscillatory phase without gravity (denoted by $\varphi_{j i}^{0}$ ). In order to measure the presence of the gravitationally induced phase, one needs to perform and compare observations on two different equipotential surfaces since the experiments on neutrino oscillations are sensitive only to the sum of both phases. To extract the full gravitationally induced phase one of these surfaces should be the surface at spatial infinity. Alternatively, it might be instructive to compare the above 
results with those of an experiment performed in a freely falling orbiter around the massive object. Specifically, the sense in which this comparison is to be performed is identical to that in which one measures a gravitational redshift of stellar spectra on Earth. The quantum-mechanically created clocks, via the time oscillation of the mass eigenstates in the linear superposition, suffer the gravitational redshift as demanded by Einstein's theory of gravitation when the gravitationally induced oscillatory phases are taken into account.

Theoretically, the prediction of Einstein's theory of gravitation as regards any clock (classically or quantummechanically driven, with a nonrelativistic or relativistic mechanism) and the prediction arising from quantum evolution that incorporates gravity via an interaction energy term are in mutual agreement for a nonrotating gravitational source. The case with a rotating gravitational source has been considered recently in Ref. [6].

In the following, the above-noted observations are appropriately modified and applied to neutrino weak-flavor eigenstates, which are empirically indicated to be linear superpositions of mass eigenstates. We again confirm the demands of Einstein's theory of gravitation in a quantum context.

\section{REDSHIFTING OF "FLAVOR-OSCILLATION" CLOCKS}

Let us assume that in the "creation region" $\mathcal{R}_{c}$ located at $\boldsymbol{r}_{c},{ }^{1}$ a weak eigenstate with energy $E$, denoted by $\left|\nu_{\ell}, \mathcal{R}_{c}\right\rangle$, is produced with the clock set to $t=t_{c}$. If each of the three neutrino mass eigenstates is represented by $\left|\nu_{i}\right\rangle, i=1,2,3$, then one is led to the linear superposition

$$
\left|\nu_{\ell}, \mathcal{R}_{c}\right\rangle=\sum_{i=1,2,3} U_{\ell i}\left|\nu_{i}\right\rangle
$$

where $\ell=e, \mu, \tau$ denotes the weak-flavor eigenstates as corresponding to electron, muon, and tau neutrinos, respectively. ${ }^{2}$ The $\left|\nu_{1}\right\rangle,\left|\nu_{2}\right\rangle$, and $\left|\nu_{3}\right\rangle$ correspond to the three mass eigenstates of masses $m_{1}, m_{2}$, and $m_{3}$, respectively. Under the already-indicated assumptions the $3 \times 3$ unitary mixing matrix $U_{\ell i}$ may be parametrized by three angles and a CP phase $\delta$ [see [8] Eq. (6.21)].

The evolution of the weak-flavor neutrino eigenstate from the creation region $\mathcal{R}_{c}$ to the "detector region" $\mathcal{R}_{d}$ corresponding to a later time $t=t_{d}>t_{c}$ and located at $\boldsymbol{r}_{d}{ }^{3}$ is given by the expression

$$
\left|\mathcal{R}_{d}\right\rangle=\exp \left(-\frac{i}{\hbar} \int_{t_{c}}^{t_{d}} H d t+\frac{i}{\hbar} \int_{\boldsymbol{r}_{c}}^{r_{d}} \boldsymbol{P} \cdot d \boldsymbol{x}\right)\left|\nu, \mathcal{R}_{c}\right\rangle
$$

\footnotetext{
${ }^{1}$ The creation region $\mathcal{R}_{c}$ is assumed to be fixed in the global coordinate system.

${ }^{2}$ Neutrinos are assumed to be of the Dirac type (for a recent analysis of various quantum field theoretic possibilities for the description of neutral particles of spin-1/2 and higher, and their relation with space-time symmetries, see Ref. [7] and references therein). In addition, we assume that both $\nu_{\ell}$ and $\nu_{m}$ are relativistic in the frame of the experimenter.

${ }^{3}$ Like the creation region, the detection region $\mathcal{R}_{d}$ is also fixed in the global coordinate system.
}

Here $H$ is the time translation operator (the Hamiltonian) associated with the system, $\boldsymbol{P}$ is the operator for spatial translations (the momentum operator), and $[H(t, \boldsymbol{x}), \boldsymbol{P}(t, \boldsymbol{x})]=0$. Consider that both $\mathcal{R}_{c}$ and $\mathcal{R}_{d}$ are located in the Schwarzschild gravitational environment [2] of a spherically symmetric object of mass $M$. The direction of neutrino propagation is along $\boldsymbol{L}=\boldsymbol{r}_{d}-\boldsymbol{r}_{c}$. In general, the state $\left|\mathcal{R}_{d}\right\rangle$ is not a definite flavor eigenstate.

As the effects of astrophysical magnetic fields and those of the interaction between the spin of the neutrino and the angular momentum of the astrophysical object are beyond the scope of the present study, we neglect the spin-dependent terms for the present. It is therefore not necessary to deal with the Dirac equation in the curved background. Semiclassical paths and the knowledge that all energy eigenstates, independent of spin, carry phases that have the general form given by Eq. (5) suffice. Under these conditions, the exponential on the right-hand side of Eq. (5) can be evaluated along the line of Stodolsky's work [9] to obtain

$$
\begin{aligned}
& \exp \left(-\frac{i}{\hbar} \int_{t_{c}}^{t_{d}} H d t+\frac{i}{\hbar} \int_{\boldsymbol{r}_{c}}^{\boldsymbol{r}_{d}} \boldsymbol{P} \cdot d \boldsymbol{x}\right)\left|\nu_{i}\right\rangle \\
& =\exp \left[-\frac{i}{\hbar} \int_{\mathcal{R}_{c}}^{\mathcal{R}_{d}}\left(\eta_{\mu \nu}+\frac{1}{2} h_{\mu \nu}\right) p_{i}^{\mu} d x^{\nu}\right]\left|\nu_{i}\right\rangle .
\end{aligned}
$$

Here $h_{\mu \nu}=g_{\mu \nu}^{W}-\eta_{\mu \nu}, g_{\mu \nu}^{W}$ is the Schwarzschild space-time metric in the weak-field limit, and $\eta_{\mu \nu}$ is the flat space-time metric. In addition, $h_{\mu \nu}=2 \phi \delta_{\mu \nu}$ with the dimensionless gravitational potential $\Phi=\phi / c^{2}=-G M / c^{2} r$. To avoid notational confusion we remind the reader that $p_{i} \equiv\left|\boldsymbol{p}_{i}\right|$; the subscript $i$ identifies the mass eigenstate and does not refer to the $i$ th component of the momentum vector.

We now calculate the "neutrino oscillation probability" from a state $\left|\nu_{\ell}, \mathcal{R}_{c}\right\rangle$ to another state $\left|\nu_{\ell^{\prime}}, \mathcal{R}_{d}\right\rangle$ following closely the standard arguments $[8,10]$, appropriately adapted to the present situation. The oscillation probability is obtained by calculating the projection $\left\langle\nu_{\ell^{\prime}}, \mathcal{R}_{d} \mid \mathcal{R}_{d}\right\rangle$, i.e., the amplitude for $\left|\nu_{\ell}, \mathcal{R}_{c}\right\rangle \rightarrow\left|\nu_{\ell^{\prime}}, \mathcal{R}_{d}\right\rangle$, and then multiplying it by its complex conjugate. An algebraic exercise that (a) exploits the unitarity of the neutrino mixing matrix $U(\theta, \beta, \psi)$, (b) exploits orthonormality of the mass eigenstates, (c) exploits certain trigonometric identities, and (d) takes care of the fact that now $d x$ and $d t$ are related by

$$
d x \simeq\left[1-\left(\frac{2 G M}{c^{2} r}\right)\right] c d t
$$

yields

$$
\begin{aligned}
\mathcal{P}\left[\left|\nu_{\ell}, \mathcal{R}_{c}\right\rangle \rightarrow \mid\right. & \left.\left|\nu_{\ell^{\prime}}, \mathcal{R}_{d}\right\rangle\right] \\
= & \delta_{\ell \ell^{\prime}}-4 U_{\ell^{\prime}} U_{\ell_{1}}^{*} U_{\ell^{\prime} 2}^{*} U_{\ell 2} \sin ^{2}\left(\varphi_{21}^{0}+\varphi_{21}^{G}\right) \\
& -4 U_{\ell^{\prime}{ }_{1}} U_{\ell 1}^{*} U_{\ell^{\prime} 3}^{*} U_{\ell 3} \sin ^{2}\left(\varphi_{31}^{0}+\varphi_{31}^{G}\right) \\
& -4 U_{\ell^{\prime} 2} U_{\ell 2}^{* L} U_{\ell^{\prime} 3}^{*} U_{\ell 3} \sin ^{2}\left(\varphi_{32}^{0}+\varphi_{32}^{G}\right) .
\end{aligned}
$$

The arguments of $\sin ^{2}()$ in the neutrino oscillation probability now contain two types of phases: the usual kinematic phase, denoted here by $\varphi_{j i}^{0}$ and defined as 


$$
\varphi_{j i}^{0} \equiv \frac{c^{3}}{4 \hbar} \frac{\left|\boldsymbol{r}_{d}-\boldsymbol{r}_{c}\right| \Delta m_{j i}^{2}}{E}=\frac{c^{3}}{4 \hbar} \frac{L \Delta m_{j i}^{2}}{E}
$$

and the new gravitationally induced phase, denoted here by $\varphi_{j i}^{G}$ and defined as

$$
\varphi_{j i}^{G} \equiv \frac{G M c}{4 \hbar} \int_{r_{c}}^{r_{d}} \frac{d l}{r} \frac{\Delta m_{j i}^{2}}{E},
$$

to first order in $\phi$ and $\Delta m_{j i}^{2}$. For variations of the gravitational potential that are small compared to the neutrino energy this integral can be approximated by

$$
\varphi_{j i}^{G}=-\langle\Phi\rangle \varphi_{j i}^{0},
$$

where $\langle\Phi\rangle$ is the average dimensionless gravitational potential over the semiclassical neutrino path

$$
\langle\Phi\rangle \equiv-\frac{1}{L} \int_{r_{c}}^{r_{d}} d l \frac{G M}{c^{2} r} .
$$

The phenomena of neutrino oscillations provides a flavoroscillation clock. Substituting the result (11) into Eq. (8), we find that the flavor-oscillation clock redshifts as required by Einstein's theory of gravitation.

\section{CONCLUDING REMARKS ON THE OBSERVABILITY OF GRAVITATIONALLY INDUCED PHASES}

In astrophysical environments $\varphi_{j i}^{G}$ may be a significant fraction of $\varphi_{j i}^{0}$. However, it is not the ratio $\varphi_{j i}^{G} / \varphi_{j i}^{0}$ alone that determines the physical relevance of the gravitationally induced phases, but also considerations of astronomical distances that are relevant to the problem at hand.

In the vicinity of a 1.4 solar mass neutron star the relevant ratio is

$$
\varphi_{j i}^{G} / \varphi_{j i}^{0} \approx 0.20
$$

Similarly,

$$
\begin{gathered}
{\left[\varphi_{j i}^{G} / \varphi_{j i}^{0}\right]_{\text {Earth }} \sim 10^{-9}, \quad\left[\varphi_{j i}^{G} / \varphi_{j i}^{0}\right]_{\mathrm{Sun}} \sim 10^{-6},} \\
{\left[\varphi_{j i}^{G} / \varphi_{j i}^{0}\right]_{\text {white dwarf }} \sim 10^{-4}}
\end{gathered}
$$

In the gravitational environment of neutron stars (and hence type-II supernovae) the neutrino oscillation probability is altered at the $20 \%$ level by the gravitationally induced phases and hence cannot be ignored under most circumstances. For the propagation of neutrinos in these environments matter effects may become important. These matter effects do not apply equally to all three flavors of neutrinos and modify the Hamiltonian in Eq. (6). The gravitationally induced phases are then proportional to the effective kinematic phases, where the masses are replaced by the effective masses in the matter. ${ }^{4}$ The relative size of the gravitational phases are thus the same as in the vacuum case. For type-II supernova the

\footnotetext{
${ }^{4}$ Again, for rapidly changing matter densities this is only true differentially and a proper integration has to be performed.
}

charged current interaction of $\nu_{e}$ is important for depositing energy to electron-flavor-rich matter. This energy deposit is dramatically modified by neutrino oscillations including their gravitational modifications if the $\Delta m_{j i}^{2}$ are such as to yield oscillations lengths that match relevant length scales for supernovae.

The effect of the gravitationally induced phases on the oscillation probabilities also depends on the number of oscillation between $\mathcal{R}_{c}$ and $\mathcal{R}_{d}$. For the gravitational field of the Sun, for example, a gravitational phase of $\pi$ is accumulated after $5 \times 10^{5}$ oscillations. Whether or not the oscillations are washed at this point depends on the energy spread and the size of the source (and the detector).

In the latest neutron interferometry experiments a discrepancy between theory and experiments continues to exist at the several standard deviation level [11]. Therefore, the question naturally arises if there exist other physical systems where the interplay of quantum mechanics and gravitation may be studied for a better understanding of the experimental systematic errors involved. Towards this end we note that it remains possible to study atomic systems that are in a linear superposition of two or more energy states in terrestrial experiments. In such systems any violation of the equivalence principle at a level of 1 part in $10^{9}$ or study of the existence of the gravivector and graviscalar fields that arise in supergravity [12] becomes experimentally accessible. $^{5}$

In summary, the basic result of this paper is as follows. The phenomenon of neutrino oscillations provides a "flavoroscillation clock." The flavor-oscillation clock redshifts as required by Einstein's theory of gravitation. Apart from the fact that these results are important for type-II supernova evolution, one may also study the interplay of the principle of equivalence and the quantum-mechanical linear superposition in atomic systems with existing technology. The integration of the gravitationally induced phase over the EarthSun distance may be relevant for the solar neutrino anomaly.

\section{ACKNOWLEDGMENTS}

We are indebted to Steven Weinberg for a comment on an earlier version of this work that led to a deeper understanding of the interplay of gravitation and quantum mechanics and Sam Werner for providing us with a copy of Ref. [11] before its publication. We thank Ephraim Fischbach for bringing to our attention Ref. [15] where gravitational effects on $K^{0}-\bar{K}^{0}$ systems have been considered. We further acknowledge useful conversations with Sam Werner and Ephraim Fischbach. Continuing discussions on space-time symmetries with V. Raatriswapan are gratefully acknowledged. This work was done, in part, under the auspices of the U.S. Department of Energy.

\footnotetext{
${ }^{5}$ After this manuscript was completed we learned of a precision atomic interferometry experiment [13] where a variation in Earth's gravity due to tides at Stanford was measured via gravitationally induced phases. Such experiments, when done with controlled local gravity sources, rather than tides, open the study of deviations [14] in the predictions based upon the principle of equivalence and the principle of linear superposition.
} 
[1] J. N. Bahcall, P. I. Krastev, and C. N. Leung, Phys. Rev. D 52, 1770 (1995); A. Halprin, C. N. Leung, and J. Pantaleone, ibid. 53, 5365 (1996); R. B. Mann and U. Sarkar, Phys. Rev. Lett. 76, 865 (1996); see also M. Gasperini, Phys. Rev. D 38, 2635 (1988); 39, 3606 (1989).

[2] S. Weinberg, Gravitation and Cosmology (Wiley, New York, 1972).

[3] L. B. Okun, Phys. Today 42 (6), 31 (1989); 43 (5), 13 (1990); 43 (5), 115 (1990); M. M. Nieto and T. Goldman, Phys. Rep. 205, 221 (1991).

[4] L. B. Okun, The Relations of Particles (World Scientific, Singapore, 1991).

[5] J. J. Sakurai, Modern Quantum Mechanics (Benjamin/ Cummings, New York, 1985), pp. 126-129. The physical considerations of this paper are in the spirit of Sakurai and his analysis of the neutron interferometry experiments. Therefore, all physical variables refer to their flat space-time values. The effects of gravity are incorporated via an interaction energy term in the quantum-mechanical equation of motion. The gravitational interaction energy term(s) are obtained as implications of the principle of equivalence and the discussion re- mains valid in the weak-field limit for both nonrelativistic and relativistic cases.

[6] D. V. Ahluwalia, Gen. Relativ. Gravit. 29, 149 (1997).

[7] D. V. Ahluwalia, Int. J. Mod. Phys. A 11, 1855 (1996); V. V. Dvoeglazov, Int. J. Theor. Phys. 34, 2467 (1996).

[8] C. W. Kim and A. Pevsner, Neutrinos in Physics and Astrophysics (Harwood Academic, New York, 1993).

[9] L. Stodolsky, Gen. Relativ. Gravit. 11, 391 (1979).

[10] H. Lipkin, Phys. Lett. B 348, 604 (1995); D. V. Ahluwalia and T. Goldman, Phys. Rev. D 56, 1698 (1997).

[11] K. C. Littrell, B. E. Allman, and S. A. Werner, Phys. Rev. A 56, 1767 (1997).

[12] J. Scherk, Phys. Lett. 88B, 265 (1979); S. Bellucci and V. Faraoni, Phys. Lett. B 392, 55 (1996).

[13] A. Peters, K. Y. Chung, B. Young, J. Hensley, and S. Chu (unpublished).

[14] C. Alvarez and R. B. Mann, Mod. Phys. Lett. A 11, 1757 (1996); S. R. Beane, Gen. Relativ. Gravit. 29, 945 (1997); J. R. Mureika, Phys. Rev. D 56, 2408 (1997).

[15] S. H. Aronson, G. J. Bock, H.-Y. Cheng, and E. Fischbach, Phys. Rev. D 28, 495 (1983). 